\title{
Supporting information for Self-constructed Multiple Plasmonic Hotspots on an Individual Fractal to Amplify Broadband Hot Electron Generation
}

Xi Wang, a, b, \# Changxu Liu, ${ }^{c, ~ \# ~ C o n g c o n g ~ G a o, ~}{ }^{a}$ Kaili Yao, ${ }^{a}$ Seyed Shayan Mousavi Masouleh, ${ }^{d}$ Rodrigo Berté, ${ }^{c}$ Haoran Ren, ${ }^{c}$ Leonardo de S. Menezes, ${ }^{c, e}$ Emiliano Cortés, ${ }^{c}$ Isobel C. Bicket, ${ }^{d}$ Haiyu Wang, ${ }^{a}$ Ning Li, ${ }^{a}$ Zhenglong Zhang, ${ }^{f}$ Ming Li, ${ }^{g}$ Wei Xie, ${ }^{h}$ Yifu Yu, ${ }^{i}$ Yurui Fang, ${ }^{j}$ Shunping Zhang, ${ }^{k}$ Hongxing Xu, ${ }^{k, 1}$ Alberto Vomiero, ${ }^{m},{ }^{n}$ Yongchang Liu, o, * Gianluigi A. Botton, ${ }^{d,}$ * Stefan A. Maier, ${ }^{c, p},{ }^{*}$ Hongyan Liang a, b, *

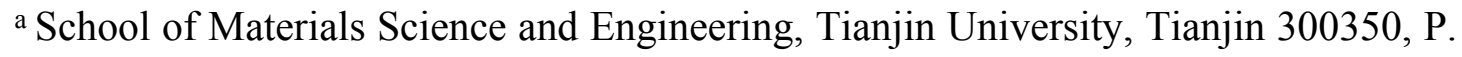
R. China

${ }^{\mathrm{b}}$ Key Laboratory of Efficient Utilization of Low and Medium Grade Energy, Ministry of Education, Tianjin University, Tianjin 300350, P. R. China.

c Chair in Hybrid Nanosystems, Nanoinstitute Munich, Faculty of Physics, LudwigMaximilians-Universität München, D-80539 München, Germany

${ }^{\mathrm{d}}$ Department of Materials Science and Engineering, McMaster University, 1280 Main Street W., Hamilton, Ontario L8S 4L7, Canada

e Leo Departamento de Física, Universidade Federal de Pernambuco, 50670-901 Recife-PE, Brazil

${ }^{\mathrm{f}}$ School of Physics and Information Technology, Shaanxi Normal University, Xi'an 710061, P. R. China 
g School of Materials Science and Engineering, State Key Laboratory for Power Metallurgy, Central South University, Changsha, Hunan 410083, P. R. China

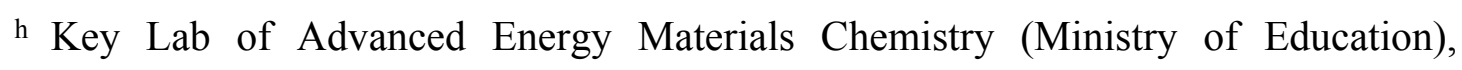
Renewable Energy Conversion and Storage Center, College of Chemistry, Nankai University, Weijin Road 94, Tianjin 300071, P. R. China

${ }^{\text {i }}$ Department of Chemistry, School of Science, Institute of Molecular Plus, Tianjin University, Tianjin, 300072, P. R. China

j Key Laboratory of Materials Modification by Laser, Electron, and Ion Beams (Ministry of Education), School of Physics, Dalian University of Technology, Dalian 116024, P. R. China

${ }^{\mathrm{k}}$ School of Physics and Technology, Center for Nanoscience and Nanotechnology, and Key Laboratory of Artificial Micro- and Nano-structures of Ministry of Education, Wuhan University, Wuhan 430072, P. R. China

${ }^{1}$ The Institute for Advanced Studies, Wuhan University, Wuhan 430072, P. R. China

${ }^{\mathrm{m}}$ Division of Materials Science, Department of Engineering Sciences and Mathematics, Luleå University of Technology, S-97187 Luleå, Sweden

${ }^{\mathrm{n}}$ Department of Molecular Sciences and Nanosystems, Ca' Foscari University of Venice, I-30172 Venezia Mestre, Italy

${ }^{\circ}$ State Key Lab of Hydraulic Engineering Simulation and Safety, School of Materials Science and Engineering, Tianjin University, Tianjin 300354, P. R. China

p Department of Physics, Imperial College London, London SW7 2AZ, England 


\section{Corresponding Author}

*E-mail: ycliu@tju.edu.cn

* E-mail: gbotton@mcmaster.ca

* E-mail: Stefan.Maier@physik.uni-muenchen.de

* E-mail: hongyan.liang@tju.edu.cn 

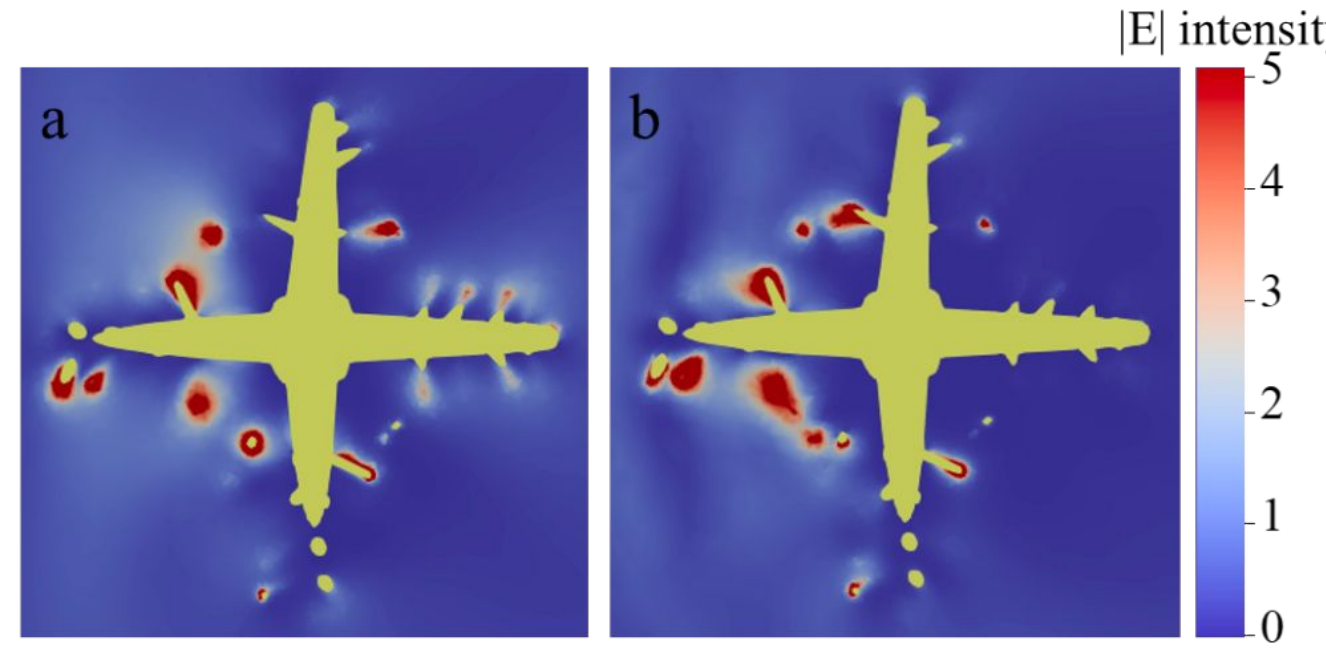

Figure S1. Simulated cross-section of the spatial distribution of intensity around an NP, upon excitation at $490 \mathrm{~nm}$ (a) and $900 \mathrm{~nm}$ (b), respectively.
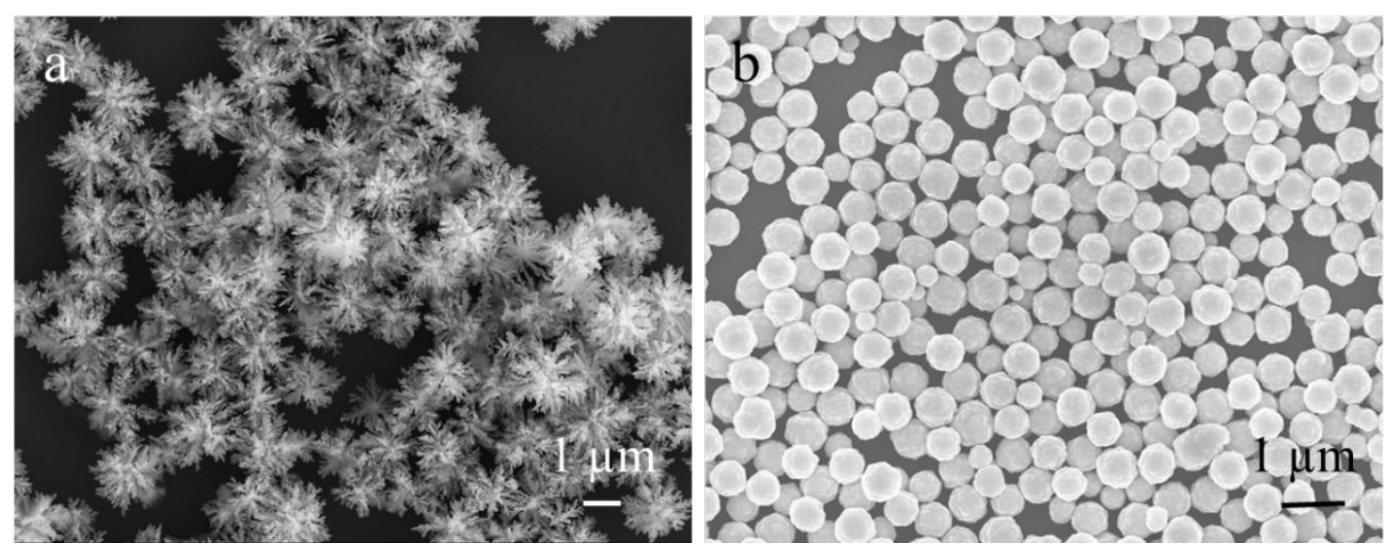

Figure S2. SEM images of silver fractal particles (a) and spheres (b). 

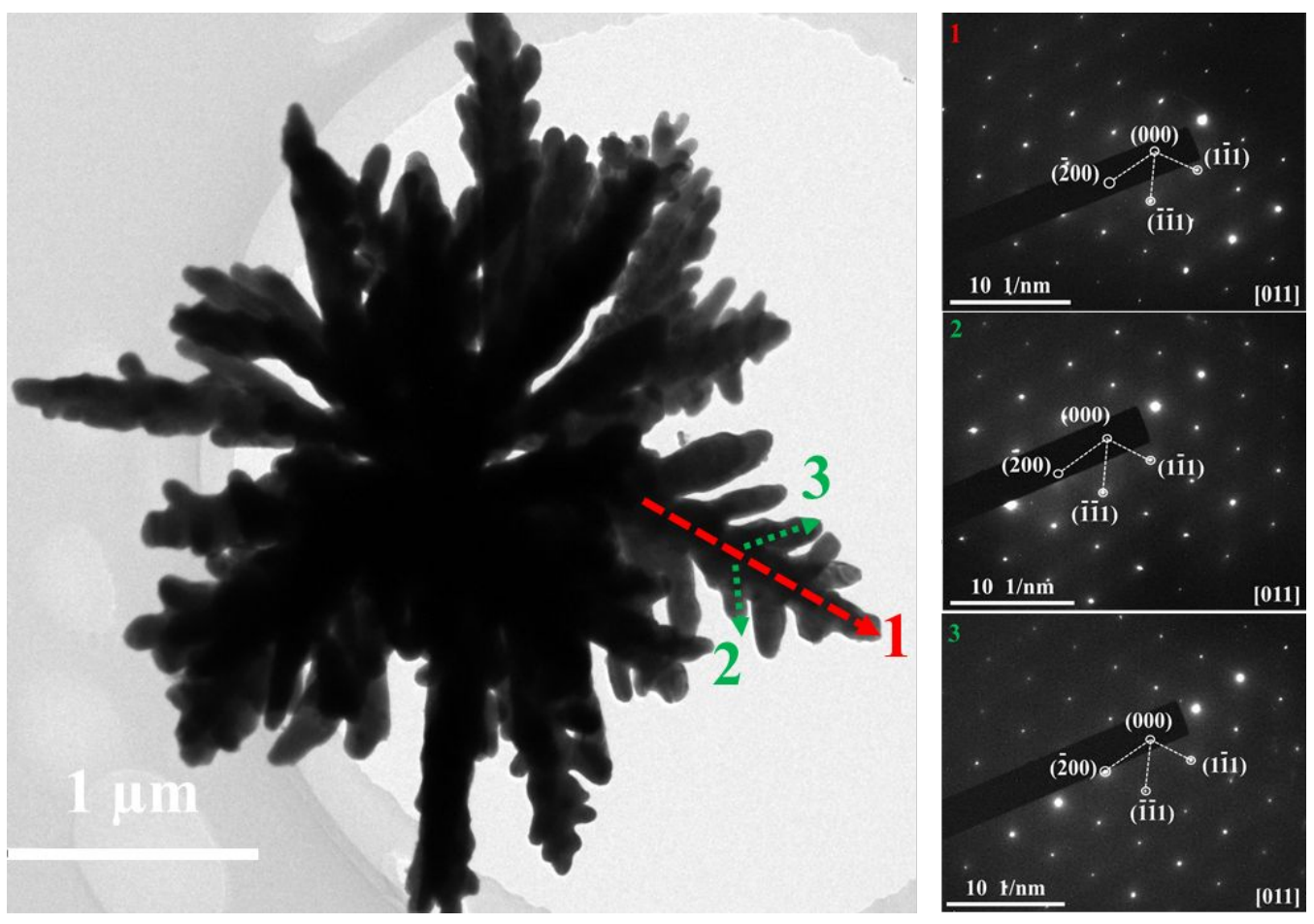

Figure S3. TEM image and SAED patterns at the tips of a silver fractal particle. All

SAED patterns were along the [011] axis of the face-centered cubic structure.

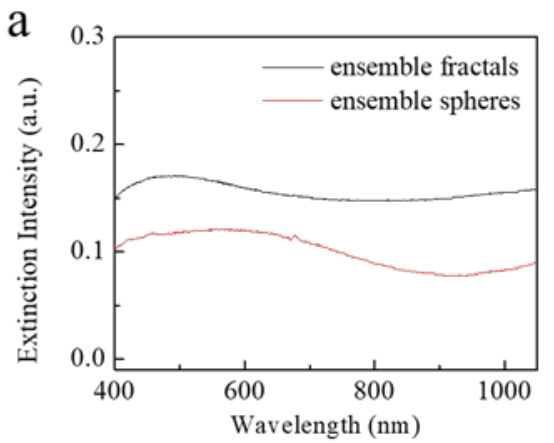

c

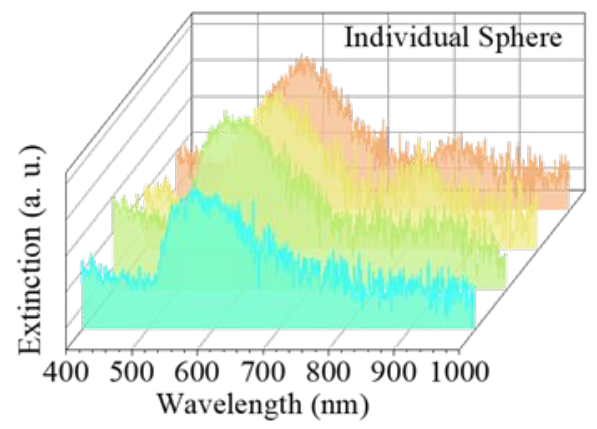

$\mathrm{b}$

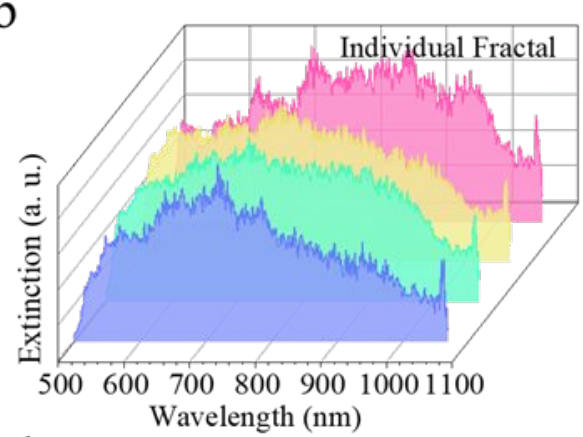

d

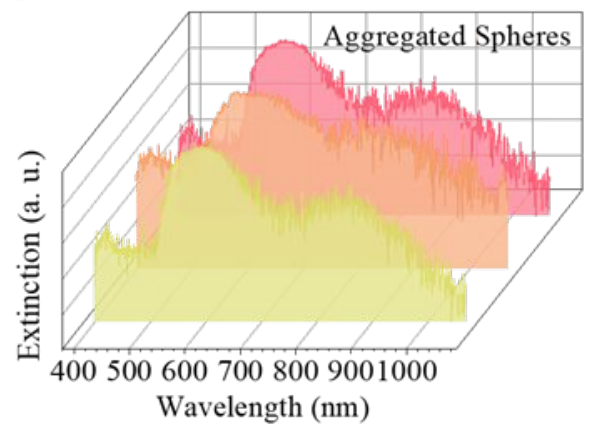


Figure S4. (a) Extinction spectra of ensemble fractals and spheres dispersed in solution characterized through the UV-vis-NIR spectrometer. Extinction spectra of four individual fractal particles (b), four individual spheres (c), and three aggregated spheres (d) deposited on glass substrate detecting by a dark field scattering mode under a confocal microscope.

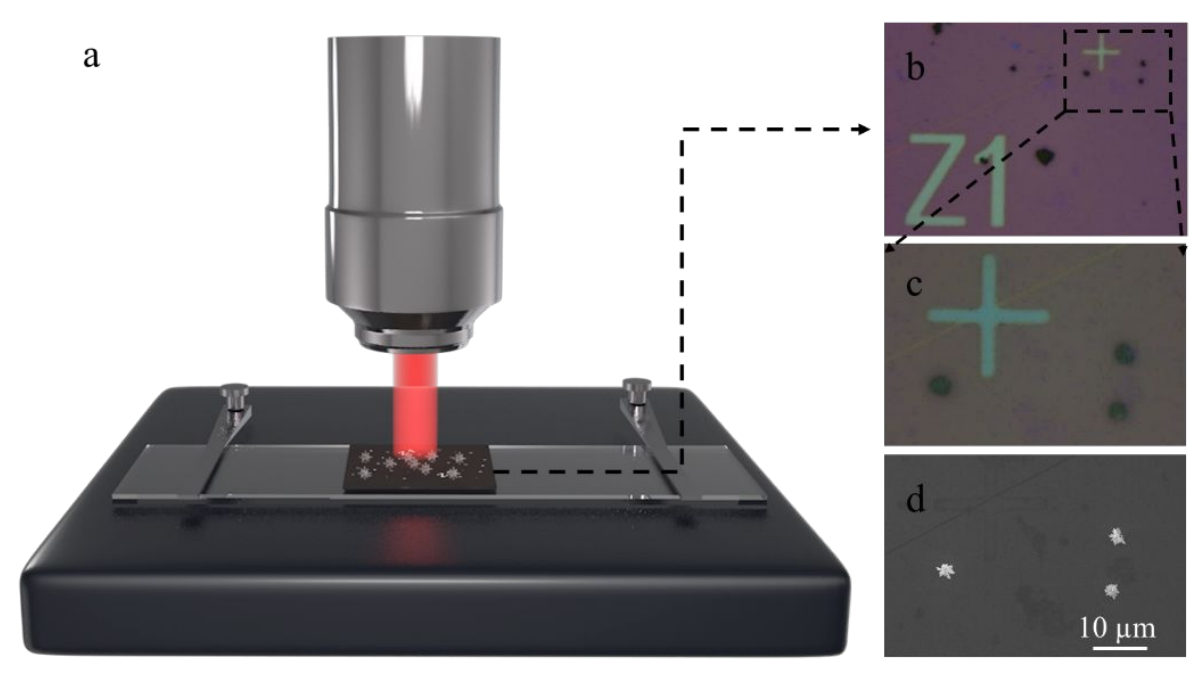

Figure S5. (a) Scheme of the set-up used for identifying the location of measured particles. (b) Low-magnification and (c) high-magnification optical image and (d) SEM image for the identified location. 

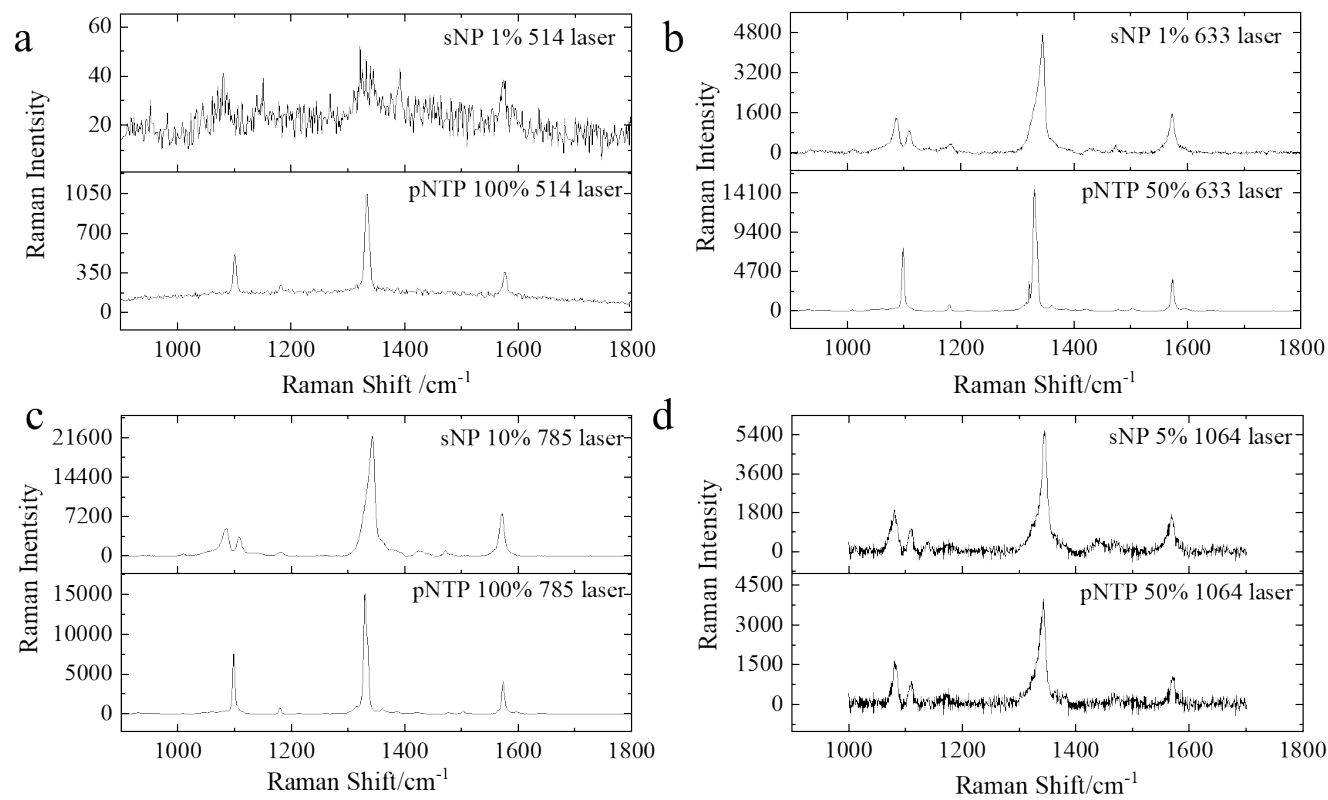

Figure S6. Typical Raman spectra for molecules absorbed on single fractal particles (sNP) and molecule powder (pNTP) obtained at different wavelengths. (a) $514 \mathrm{~nm}$, (b) $633 \mathrm{~nm}$, (c) $785 \mathrm{~nm}$ and (d) $1064 \mathrm{~nm}$.

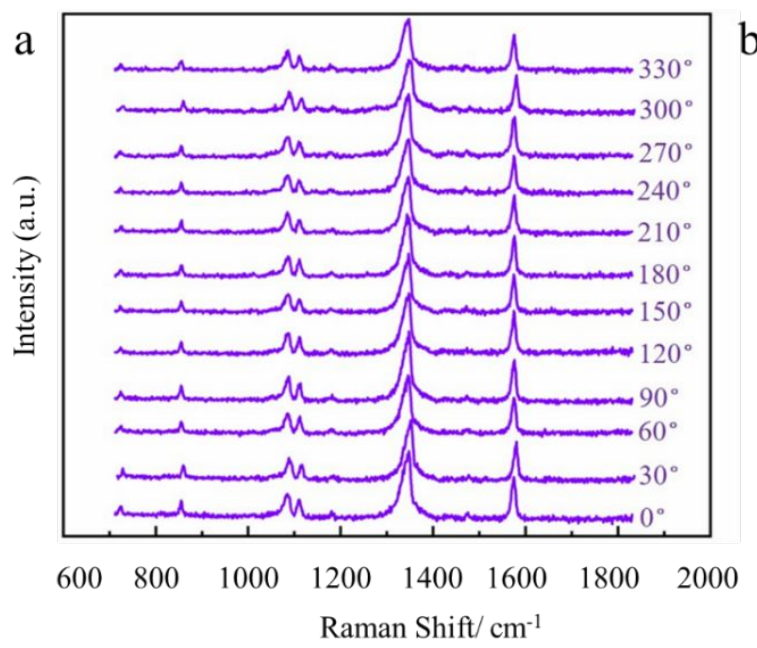

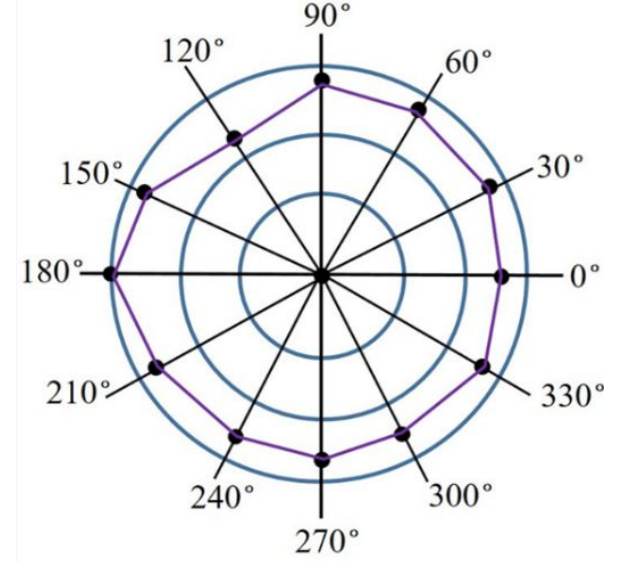

Peak intensity at $1346 \mathrm{~cm}^{-1}$

Figure S7. Investigation of the behavior of polarization-dependent SERS on the single fractal particle by changing the laser incidence angle from 0 to $360^{\circ}$. Raman spectra (a) and peak intensity (b) at $1346 \mathrm{~cm}^{-1}$ under excitation at various polarization angles. The 
SERS keeps nearly identical intensity regardless of the incident polarization, indicating polarization-independent plasmonic feature.
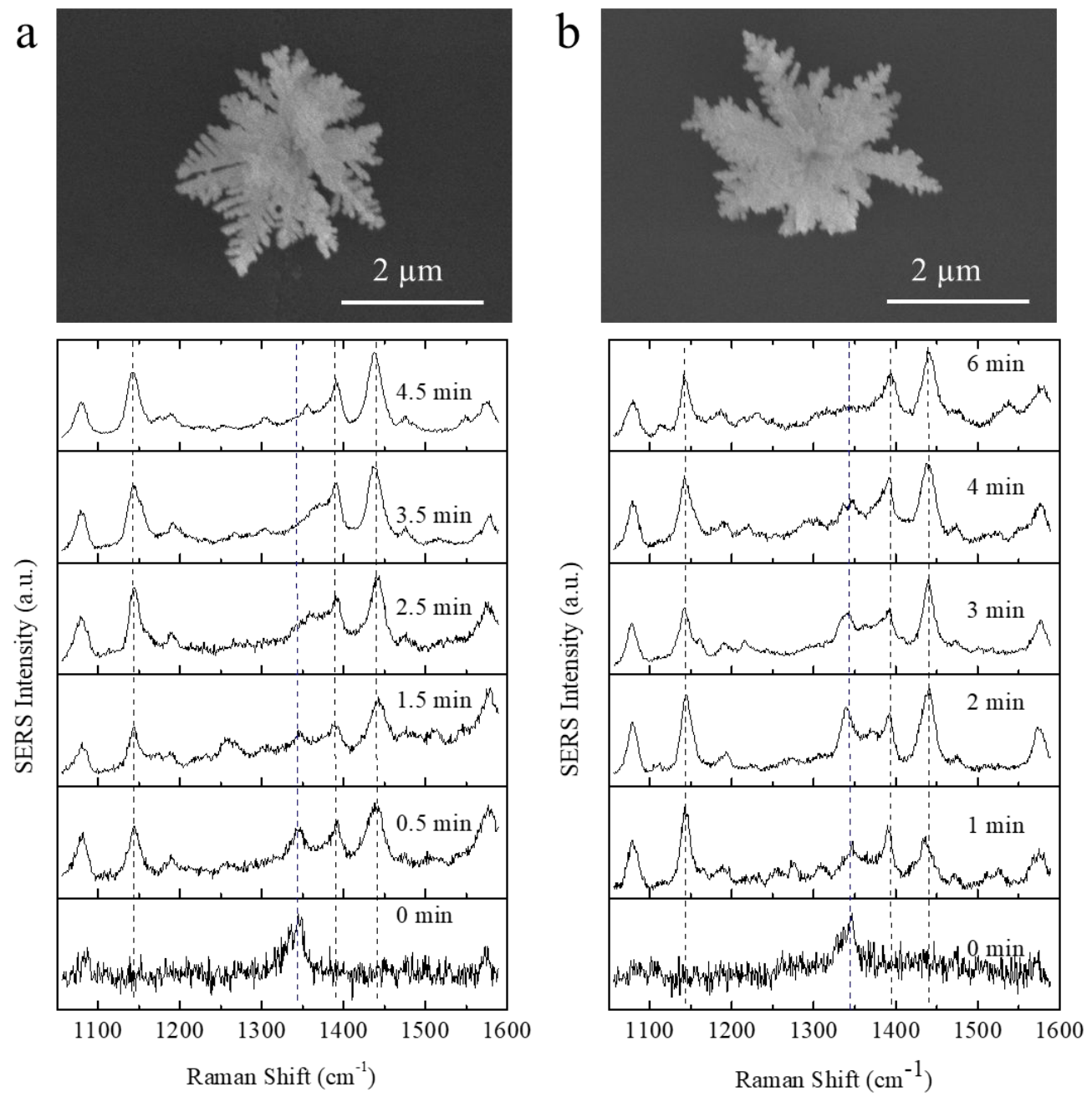

Figure S8. (a) and (b) SEM images and SERS spectra on single fractal particle after irradiation for different time with 633 nm laser. The characteristic Raman bands at 1346 and $1143,1390,1440 \mathrm{~cm}^{-1}$ are assigned to $p$ NTP (blue vertical dash line) and DMAB (black vertical dash line), respectively. 

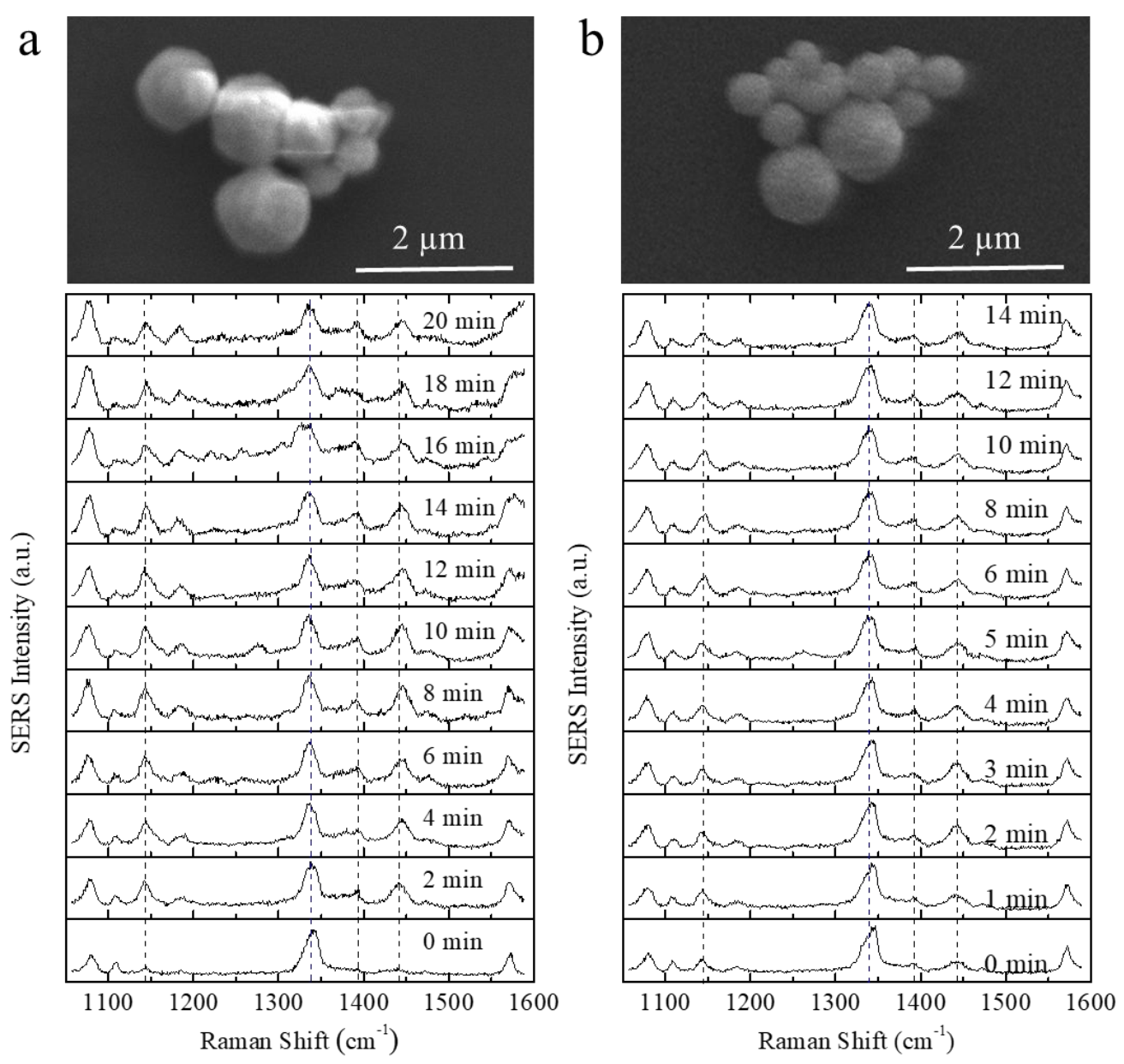

Figure S9. (a) and (b) SEM images and SERS spectra on aggregated spheres after irradiation for different time with $633 \mathrm{~nm}$ laser. The characteristic Raman bands at 1346 and $1143,1390,1440 \mathrm{~cm}^{-1}$ are assigned to $p$ NTP (blue vertical dash line) and DMAB (black vertical dash line), respectively. 


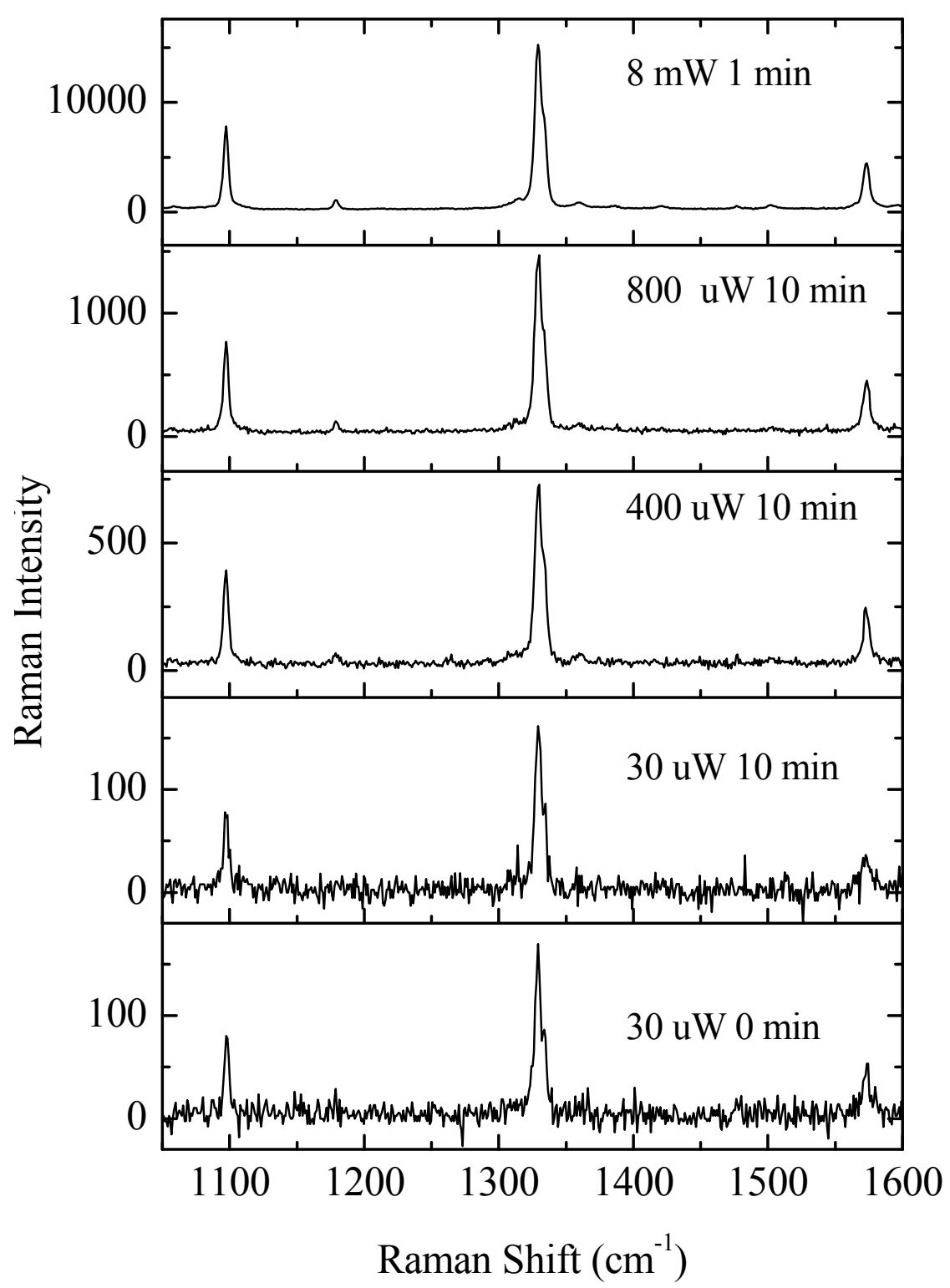

Figure S10. Raman spectra of pure $p$ NTP molecules after irradiation with $633 \mathrm{~nm}$ laser for different time with varied laser power. No reduction happens without the presence of silver structures due to the lack of hot electrons. 

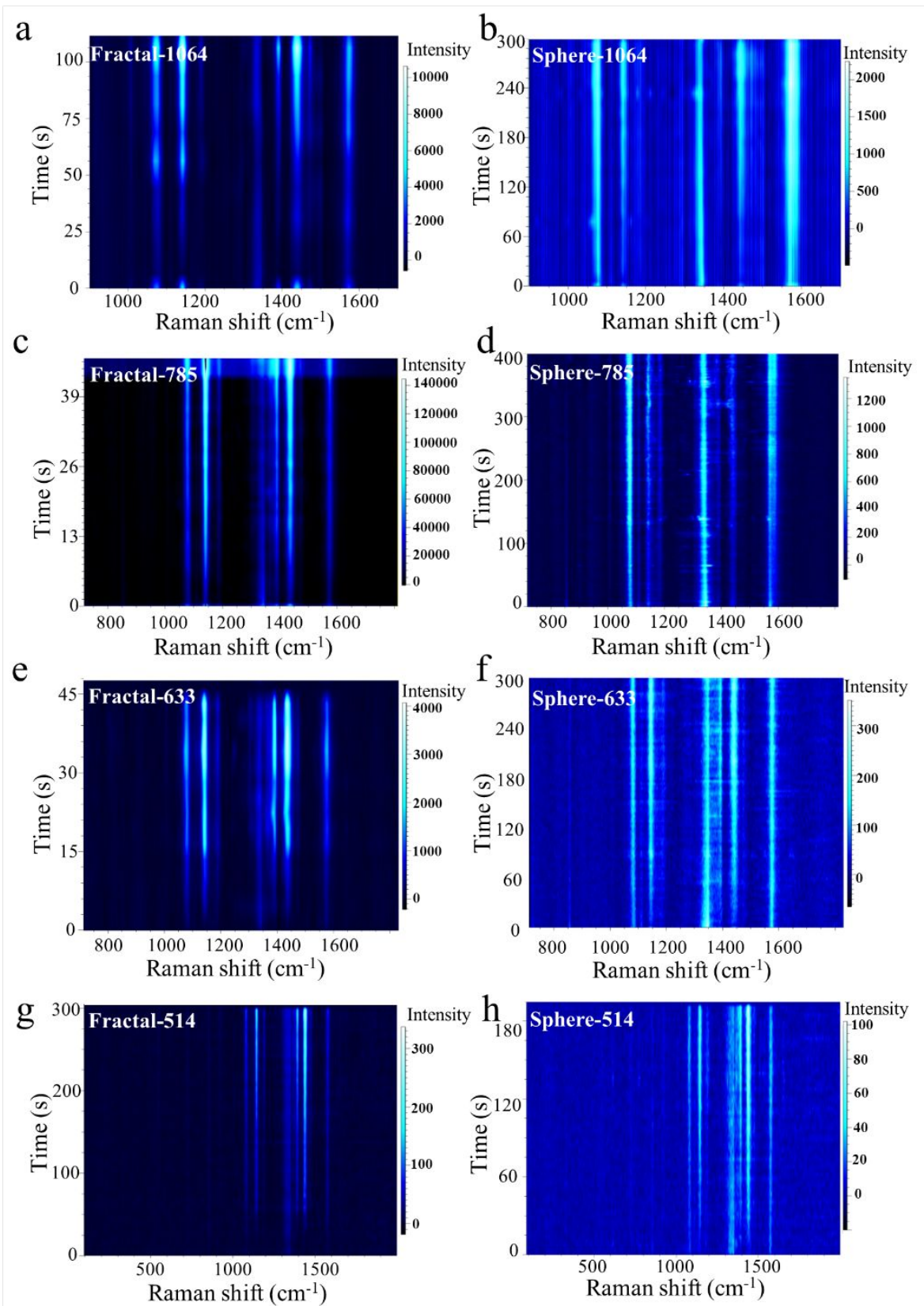

Figure S11. Spectro-temporal Raman scattering measurements monitoring the reaction process on the surface of the single fractal particle and aggregated spheres under different excitation wavelengths. (a, b) $1064 \mathrm{~nm}$, (c, d) $785 \mathrm{~nm}$, (e, f) $633 \mathrm{~nm}$ and (g, h) $514 \mathrm{~nm}$. 

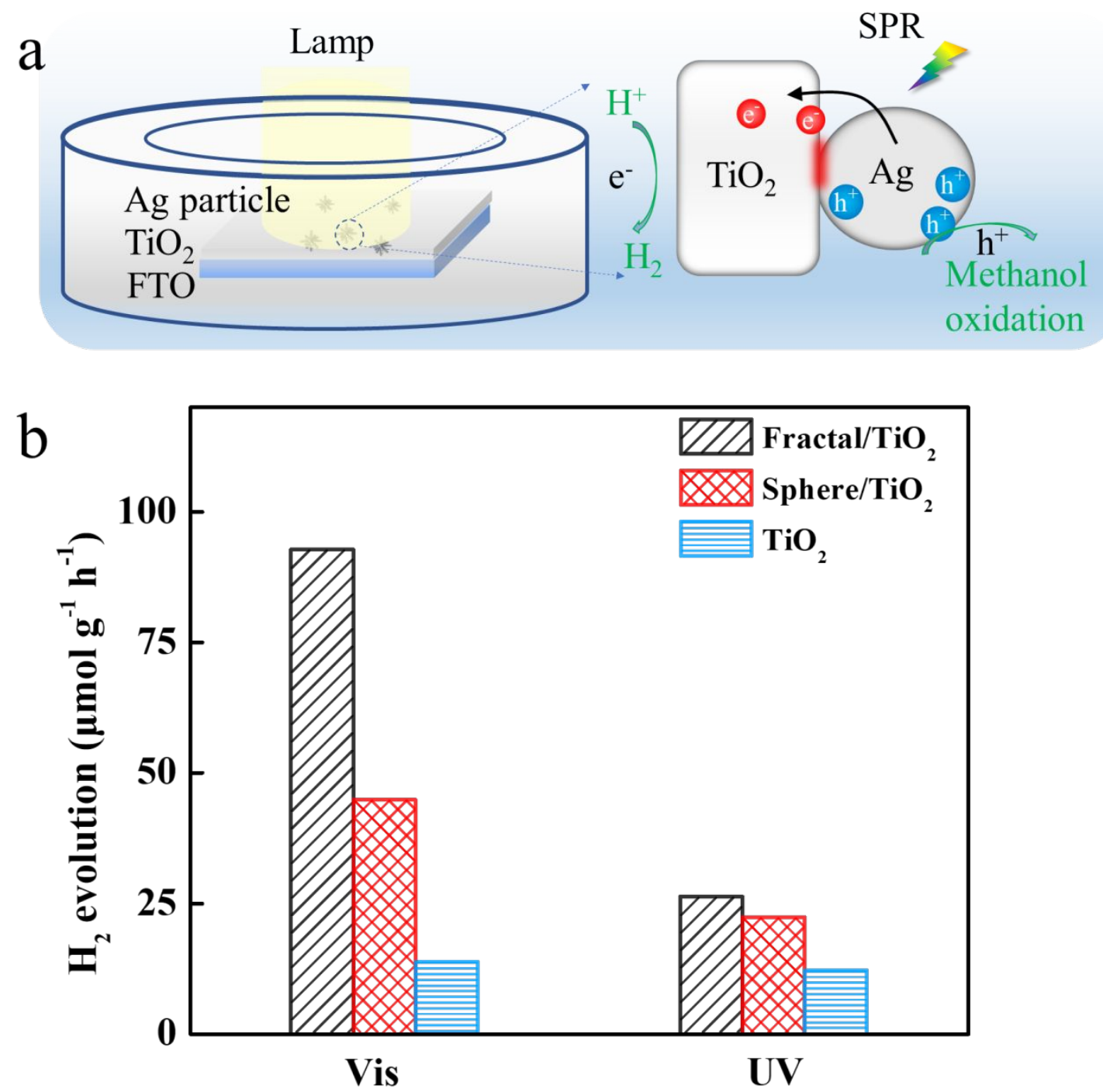

Figure S12. (a) Schematic illustration of the setup (left) and the proposed mechanism for plasmon induced electron transfer for photocatalysis. (b) Hydrogen generation rates for visible $\left(\lambda>420 \mathrm{~nm}, 300 \mathrm{mw} / \mathrm{cm}^{2}\right)$ and $\operatorname{UV}\left(\lambda=260-380 \mathrm{~nm}, 9.05 \mathrm{~mW} / \mathrm{cm}^{2}\right)$ illumination. 
Table S1: Experimental parameters for SERS detection and enhancement factor (EF) calculation

\begin{tabular}{|l|l|l|l|l|l|}
\hline $\begin{array}{l}\text { Laser } \\
\text { wavelength } \\
(\mathrm{nm})\end{array}$ & $\begin{array}{l}\text { Laser } \\
\text { diameter } \\
D(\mathrm{~nm})\end{array}$ & $\begin{array}{l}\text { Focus depth } \\
f(\mathrm{~nm})\end{array}$ & $N_{\text {bulk }}$ & $N_{\text {SERS }}$ & $\begin{array}{l}\text { EF for single } \\
\text { fractal particle }\end{array}$ \\
\hline 514 & 1,254 & 5,201 & $3.3 \times 10^{10}$ & $7.5 \times 10^{5}$ & $0.9 \times 10^{6}$ \\
\hline 633 & 1,544 & 6,406 & $6.3 \times 10^{10}$ & $7.5 \times 10^{5}$ & $1.5 \times 10^{6}$ \\
\hline 785 & 1,915 & 7,944 & $1.2 \times 10^{11}$ & $7.5 \times 10^{5}$ & $2.3 \times 10^{7}$ \\
\hline 1064 & 2,596 & 10,768 & $3.0 \times 10^{11}$ & $7.5 \times 10^{5}$ & $6.6 \times 10^{6}$ \\
\hline
\end{tabular}

Table S2: Values of $k\left(\mathrm{~s}^{-1}\right)$ for both kinds of samples studied as a function of the excitation wavelength

\begin{tabular}{|c|c|c|c|c|}
\hline \multirow{2}{*}{ Sample } & \multicolumn{4}{|c|}{ Excitation wavelength $(\mathrm{nm})$} \\
\cline { 2 - 5 } & 514 & 633 & 785 & 1064 \\
\hline Fractal particle & $0.225 \pm 0.004$ & $0.264 \pm 0.08$ & $0.151 \pm 0.03$ & $0.365 \pm 0.04$ \\
\hline Spheres & $0.043 \pm 0.01$ & $0.025 \pm 0.007$ & $0.005 \pm 0.0008$ & $0.014 \pm 0.005$ \\
\hline
\end{tabular}

